\title{
Über die Bestimmung des Wassers in Butter und Margarine nach dem „Aluminiumbecher-Verfahren“.
}

\author{
Von \\ G. Fendler und W. Stiiber.
}

Mitteilung aus dem Untersuchungsamteder Stadt Berlin.

Neuerdings werden verschiedentlich gewichtsanalytische Schnellmethoden $\mathrm{zur}$ Bestimmung des Wassergehaltes der Butter empfohlen, welche daranf beruhen, da das Wasser nicht im Trockenschranke, sondern über freier Flamme verdunstet wird.

Nach H. Droop Richmond ) erhitzt man etwa $10 \mathrm{~g}$ Butter unter Umrübren mit einem Glasstabe in einer Porzellanschale. G. E. Patrick ${ }^{2}$ ) erhitzt. 12-16 $\mathrm{g}$ Butter in einem Reagensglase von $19 \mathrm{~cm}$ Länge und $3,8 \mathrm{~cm}$ Weite. Die Abweichungen von exakten Bestimmungen sollen nach diesem Verfahren meist nur etwa $0,1 \%$, selten mehr als $0,3 \%$ betragen. Derselbe Verfasser ${ }^{3}$ ) beschreibt eine Methode, nach der $10 \mathrm{~g}$ Butter in einem Aluminiumbecher unter stetem Umschwenken erhitzt werden sollen. Nach einem Flugblatt der schwedischen "Zentralanstalt für landwirtschaftliche Versuch e*4) liefert die sogenannte englische Methode (Droop Richmond'sche Methode) „überraschend gleichmäßige und gute Resultate". Die niedrigste beobachtete Differenz gegenüber der offiziellen (Trockenschrank-) Methode betrug $0,04 \%$, die höchste $0,37 \%$, in den meisten Fällen machte sie $0,17 \%$ aus. Infolge der guten Ergebnisse einpfiehlt die genannte Zentralanstalt die „englische Methode“ zur Anwendung in den Meiereien. Benutzt werden Nickelschalen. Die Wägungen werden auf einer besonders zu diesem Zwecke konstruierten Wage ausgeführt.

A. Wingler und Jos. von Suryã) beschreiben einen von F. W. Nicholls in London zusanmengestellten ,Sensible-Apparat", dessen Hauptteile aus einer Wage und einem Aluminiumbecher zum Erhitzen der Butter bestehen. Angewendet werden etwa $11 \mathrm{~g}$ Butter. Die Verfasser beobachteten eine Höchstdifferenz von $0,22 \%$ gegenüber der Trockenschrankmethode.

Kürzlich lernten wir einen ähnlichen, von einer deutschen Firma in den Handel gebrachten Apparat „Perplex"6) kennen. Fs schien uns der Mühe wert, das „englische Verfahren" an der Hand dieses Apparates nachzuprüfen, denn der Wert einer wirklich genauen Schnellmethode ist in vielen Fällen nicht zu verkennen.

Der Apparat "Perplex" besteht aus einer einarmigen Wage, einem Aluminiumbecher von etwa $170 \mathrm{ccm}$ Fassungsvermögen und etwa $38 \mathrm{~g}$ Gewicht, sowie der zum Halten des Bechers bestimmten Zange.

1) Vergl. Harald Faber, Maelkeritidende, 1907, 20, 199-211; Milchwirtschaftl. Zentralbl. $1908,4,9$.

2) Journ. of the Americ. Chem. Soc. 1906, 28, 1611 ; Milchwirtschaftl. Zentralbl. 1908, 4, 40.

3) New York Produce Review and American Creamery 1907, 24, 638; Milchwirtschaft1. Zentralbl. 1908, 4, 282.

4) Norsk Landmandsblad, 27, 242-244; Milchwirtschaftl. Zentralbl. 1908, 4, 475.

5) Diese Zeitschrift 1908, 16, 403.

") „Perplex", Funke's neuer Untersuchungsapparat zur Feststellung des Wassergehaltes der Butter. - Der Apparat ist zu beziehen von Paul Funke \& Co., Berlin $N$, Chausseegtrafie 10). 
Der mit $10 \mathrm{~g}$ Butter beschickte Becher wird unter Benutzung der Zange und unter stetem Umschwenken über einer kleinen Flamme erhitzt. Bis zum völligen: Verjagen des Wassers sind bei Butter etwa 4 Minuten notwendig.

In dem Prospekte der Firma werden dafür, daß das Wasser völlig verdunstet ist, folgende Merkmale angegeben:

1. Gänzliches Aufhören des Knisterns,

2. Bildung eines weißen Fettschaumes, welcher mit dem Aufhören des Knatterns zusammenfällt,

3. der angenehme Geruch, welcher sich beim Bräunen der Butter zeigt.

4. die beginnende Bräunung der Kaseinbestandteile.

Diese Anhaltspunkte genügen jedoch nach unseren Erfahrungen nicht; es ist vielmehr notwendig, gegen Ende der Operation durch öfteres Auflegen eines kalten Uhrglases auf den Becher festzustellen, ob noch Wasser verdunstet. So lange dies der Fall ist, bedeckt sich das Uhrglas mit einem Beschlag. Überhitzt man, so bilden sich infolge Zersetzung der Butterbestandteile deutlich sichtbare Dämpfe, welche mit Wasserdämpfen kaum zu verwechseln sind. Hat man die Erhitzung versehentlich bis zu diesem Punkte getrieben, unterbricht sie jedoch sofort beim Auftreten der Dämpfe, so ist der Fehler nur ein sehr geringer. Nach Angabe der Firma werden dann etwa $4 / 10 \%$ Wasser zu viel gefunden; nach unseren Beobachtungen war die Differenz noch kleiner.

Der erhitzte Tiegel wird nach völligem Erkalten zurückgewogen; unrichtig ist es, ihn laut Prospekt schon nach 3/4 Minuten zu wägen, wodurch Abweichungen von mehreren Zehntelprozenten entstehen können.

Die Ergebnisse, welche wir mit 11 Butterproben des Handels erhielten, sind in Tabelle I zusammengestellt.

Tabelle I. Butter.

\begin{tabular}{|c|c|c|c|c|}
\hline \multirow[b]{2}{*}{$\begin{array}{l}\text { Butter } \\
\text { No. }\end{array}$} & \multirow[b]{2}{*}{$\begin{array}{c}\text { W } \\
\text { Wasserbestimnung im } \\
\text { Sox hiet'sehen Glyec- } \\
\text { rin-Troekensehranke bei } \\
105^{\circ} \mathrm{O} \\
0_{i 0}^{\prime}\end{array}$} & \multicolumn{3}{|c|}{ Wasserbestimmung nach dem Aluminiumbecher-Verfahren } \\
\hline & & $\begin{array}{l}\text { Der Becher naeh volli- } \\
\text { gem Erkalten zurückge- } \\
\text { wogen } \\
\%\end{array}$ & $\begin{array}{c}\text { Der Becher nach } 3 / 4 \mathrm{Mi} \text { - } \\
\text { nuten zurüukgew ogen } \\
\%\end{array}$ & $\begin{array}{c}\text { d } \\
\text { Bis zur beginnenden } \\
\text { Zersetzung(Bildung von } \\
\text { Damplen erhitzt } \\
\%\end{array}$ \\
\hline 1 & 18,60 & 18,$65 ; 18,70 ; 18,55$ & 18,80 & 18,80 \\
\hline 2 & 14,98 & 14,$90 ; 14,90$ & 15,$15 ; 15,10$ & 14,95 \\
\hline 3 & 14,75 & 14,$70 ; 14,65$ & 15,$00 ; 14,85$ & 14,$90 ; 14,80$ \\
\hline 4 & 14,12 & 14,$05 ; 14,10$ & - & 14,$15 ; 14,20$ \\
\hline 5 & 13,39 & 13,$50 ; 13,50$ & - & - \\
\hline 6 & 14,52 & 14,50 & - & - \\
\hline 7 & 14,72 & 14,75 & - & - \\
\hline 8 & 14,31 & 14,$40 ; 14,40$ & - & 14,50 \\
\hline 9 & 13,23 & 13,20 & - & - \\
\hline 10 & 14,00 & 14,15 & - & - \\
\hline 11 & 14,16 & 14,$05 ; 14,10$ & - & - \\
\hline
\end{tabular}


Aus' Spalte.b dieser Tabelle ist ersichtlich, daß bei Doppelbestimmungen nennenswerte Abweichungen nicht erhalten werden. Ein Vergleich von Spalte a und b zeigt ferner, daß die größte Abweichung von der Trockenschrankmethode $0,15 \%$ beträgt. Erhitzt man zu stark, so können (siehe Spalte d) Differenzen bis zu $0,2 \%$ vorkommen. Aus den Zahlen der Spalte c geht hervor, daß der Becher zweckmäßig erst nach völligem Erkalten zurückgewogen wird.

Dieses Verfahren liefert somit sehr gute Ergebnisse; seine Anwendung kannzur schnellen und genauen Ermittelung des Wassergehaltes der Butter empfohlen werden.

Wir nahmen die Wăgungen mit der dem Apparat beigegebenen Wage vor, von deren Genauigkeit wir uns überzengt hatten. Selbstverständlich tut jede andere analytische Wage dieselben Dienste.

Wir dehnten unsere Versuche ferner auch auf Marga rin e-Proben des Handels aus. Margarine verhält sich bekanntlich beim Erhitzen nicht immer wie Naturbutter, sondern neigt leichter zum Spritzen als diese.

Die in Tabelle II zusammengestellten Ergebnisse zeigen, daf sich im allgegemeinen auch in der Margarine der Wassergehalt recht gut nach dem Aluminiumbecher-Verfahren bestimmen läßt. Es gibt aber auch Margarinesorten, auf welche dies nicht zutrifft. Eine sogenannte "Konditormargarine" mit geringem Wassergehalte (etwa 6,5\%) spritzte beim Erhitzen im Aluminiumbecher so stark, daß der Versuch abgebrochen werden mußte. Derartige Margarineproben dürfen natürlich nicht nach diesem Verfahren untersucht werden.

Tabelle II. Margarine.

\begin{tabular}{|c|c|c|c|c|}
\hline \multirow[b]{2}{*}{$\begin{array}{c}\text { Marga- } \\
\text { rine } \\
\text { No. }\end{array}$} & \multirow[b]{2}{*}{$\begin{array}{l}\text { a } \\
\text { Wasserbestimmung im } \\
\text { Soxhl et'schen Glyce- } \\
\text { rin-Trockensthranke bei } \\
105^{\circ} \mathrm{C} \\
\%\end{array}$} & \multicolumn{3}{|c|}{ Wasserbestimmung nach dem Aluminiumbecher-Verfahren } \\
\hline & & $\begin{array}{c}\text { b } \\
\text { Der Bechor naeh völl- } \\
\text { gen Erkalten zurtiekge- } \\
\text { wogen } \\
\text { \% }\end{array}$ & 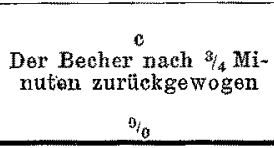 & $\begin{array}{c}\text { d } \\
\text { Bis zur beginuenden } \\
\text { Zersetzung (Bildang von } \\
\text { Dämpfen) erhitzt } \\
0 / 6\end{array}$ \\
\hline 1 & 10,83 & 10,$90 ; 10,80$ & 11,$05 ; 11,00$ & 11,$00 ; 10,95$ \\
\hline 2 & 14,94 & 14,$90 ; 14,90$ & 15,05 & 15.10 \\
\hline 3 & 15,25 & 15,40 & 15,60 & 15,60 \\
\hline 4 & 10,76 & 10,70 & - & 10,85 \\
\hline 5 & 16,59 & 16,$60 ; 16,60$ & - & - \\
\hline 6 & 16,58 & 16,$55 ; 16,60$ & - & 一 \\
\hline 7 & 15,45 & 15,$55 ; 15,50$ & - & 15.65 \\
\hline 8 & 16,45 & 16,50 & - & 16,65 \\
\hline
\end{tabular}

